\title{
Algunos aspectos cuantitativos sobre la relación entre la emigración internacional y la migración interna de mexicanos
}

\author{
Rodolfo Corona Vázquez \\ Universidad Nacional Autónoma de México
}

\begin{abstract}
Resumen
Este trabajo pretende establecer algunas relaciones entre los determinantes y las consecuencias de la emigración de mexicanos a Estados Unidos y de la migración al interior de la República Mexicana. Se realiza un análisis comparativo de información estadística (censos, encuestas, registros, etc.) acerca de las características económicas, sociales y geográficas de los migrantes internos, de los emigrantes internacionales y de los que son internos e internacionales al mismo tiempo. Con este análisis se pretende identificar e interpretar similitudes y diferencias, para explicar el porqué algunos mexicanos han optado, o se han visto forzados a desplazarse dentro de los límites territoriales de la nación, hacia el exterior o hacia ambas partes.
\end{abstract}

Palabras clave: emigración internacional, migración interna, población, desplazamiento humano, indocumentados.

\begin{abstract}
The purpose of this work is to establish some existing relations between the determining factors and the consequences of Mexican immigration of Mexican workers to the United States, and the migration to the interior of the Mexican Republic. A comparative analysis on statistic information is performed (census, surveys, records, etc.) specifically about economic, social, and geographical characteristics of the country migrants, of the international migrants, and those who are both country and international migrants. Through this analysis we pretend to identify and interpret the similarities and differences, to explain why some Mexican have decided or are forced to move within the boundaries of the national territory, or beyond the borders, and some times they move into both directions.
\end{abstract}

Keywords: international migration, internal migration, population, human displacement, undocumented. 


\title{
ALGUNOS ASPECTOS CUANTITATIVOS SOBRE LA RELACION ENTRE LA EMIGRACION INTERNACIONAL Y LA MIGRACION INTERNA DE MEXICANOS
}

\author{
Por \\ Rodolfo Corona Vázquez \\ Investigador del Instituto de Investigaciones \\ Sociales de la Universidad Nacional \\ Autónoma de México
}

\section{INTRODUCCION}

Este trabajo forma parte de un proyecto de investigación más amplio donde se pretenden establecer algunas relaciones entre los determinantes y las consecuencias de la emigración de mexicanos a Estados Unidos y de la migración al interior de la República Mexicana. ${ }^{1}$

Tal proyecto se desarrolla abordando paralelamente dos grandes líneas de acción que se entrecruzan y complementan: de una parte, la línea que hemos llamado teórica y que consiste en confrontar las teorías explicativas de ambos fenómenos a fin de encontrar puntos de conexión y de alejamiento; de otra parte, el camino de naturaleza empírica, que se conforma por un análisis comparativo de la información estadística existente (producto de censos, encuestas por muestreo y registros administrativos) acerca de las características economicas, demográficas, sociales y geográficas de los migrantes internos, de los emigrantes internacionales y de aquellos que son internos e internacionales al mismo tiempo. Este análisis comparativo de datos tiene el propósito de identificar y tratar de interpretar similitudes y diferencias, tanto para dar explicaciones sobre el porque algunos mexicanos optan o se ven forzados a desplazarse dentro de los límites territoriales de la nación, hacia el exterior o hacia ambas partes, como para apoyar o poner en tela de juicio los resultados de la investigación de naturaleza teórica.

El presente trabajo forma parte de la vertiente empírica del citado proyecto, es decir, se refiere exclusivamente a la comparación de las características cuantificadas de los migrantes internos y de los emigrantes a Estados Unidos; con los resultados de esta comparación se intenta iniciar la

\footnotetext{
${ }^{1}$ Eate proyecto, denominado "Relaciones entre la migración interna y la internacional", se desarrolla conjuntamente por los investigadores Rodolfo Corona Vázquez (Instituto de Investigaciones Sociales de la Universidad Nacional Autónoma de México) y Crescencio Ruiz Chiapet to (Centro de Estudios Económicos y Demográficos de El Colegio de México). Hasta la fecha, dentro de este proyecto se han realizado dọs estudios:

"Migración Interna e Internacional: algunos resultados de la Encuesta Nacional de Emigración a la Frontera Norte del País y aslos Estados Unidos". Ponencia presentada por R. Corona y C. Ruiż al Simposio Na. cional sobre Emigración y Distribución Espacial de la Población, efectuado en Guadalajara, Jalisco en diciembre de 1979 .

"Migrantes Internacionales con y sin antecedentes de migración interna: algunas características socioeconómicas". Trabajo elaborado por R. Corona y C. Ruiz para el Centro Nacional de Información y Estadísticas del Trabajo, de la Secretaría del Trabajo y Previsión Social; México, Julio de 1980.
} 
búsqueda de elementos de hipótesis acerca de la vinculación entre los fenó menos de migración interna e internacional.

El trabajo se compone por tres apartados, además de las conclusione: y de esta breve introducción. En el primer apartado se habla de los proble mas prácticos inherentes a un estudio de esta naturaleza y se delimitan las poblaciones básicas objeto de estudio (los emigrantes internacionales divi

didos entre aquellos que no tienen antecedentes de migración interna, emigrantes internacionales "puros" y aquellos que tienen experiencias de migración interna); en el segundo se realiza un análisis del porcentaje que representa el grupo de personas que son al mismo tiempo migrantes internos y emigrantes a Estados Unidos con respecto a los totales de migrantes internos y de emigrantes internacionales "puros", usando para ello distintas definiciones de migración interna; y en el tercer apartado se comparan ciertas características (demográficas, sociales, económicas y geográficas) de los tres tipos de migrantes mencionados.

\section{LA POBLACION OBJETO DE ESTUDIO:}

Para llevar a cabo un análisis comparativo de las características socioeconómicas, geográficas y demográficas de los tres tipos de migrantes: migrantes internos, migrantes internacionales "puros" y migrantes internacionales con experiencia de migración interna, incluyendo la medición de sus volúmenes, se presentan varios problemas prácticos, siendo los primeros a resolver los de orden conceptual y los de existencia de información numérica adecuada. Es decir, para comparar la cantidad y las características de distintos grupos de migrantes resulta necesario contar con las definiciones de cada uno de ellos, además de contar con datos confiables y relacionables al respecto. Ambos requerimientos se convierten en problemas para el caso de México por dos razones.

En primer lugar, porque tanto las migraciones internas como las internacionales se componen por muy variadas clases de desplazamientos, por ejemplo, hablando de las migraciones internas, se sabe que existen movimientos migratorios de carácter definitivo, temporales y estacionales, los cuales, amén de combinarse, involucran desplazamientos entre estados, municipios o localidades, y de zonas rurales a urbanas, de urbanas a rurales, entre áreas rurales o bien entre ciudades; asimismo, estos movimientos se realizan por individuos solos y por familias. Hablando de las migraciones de mexicanos a Estados Unidos, también se tiene conocimiento de que adquieren distintas formas: legales y de trabajadores sin documentos, definitivas $\mathrm{y}$ temporales, individuales y familiares, etc.

En segundo lugar, el problema surge por la falta de información estadística que documente tanto los movimientos migratorios internos como los internacionales. En cuanto a los primeros, únicamente se dispone de los censos de población que dan cuenta de migraciones internas definitivas entre 
entidades federativas, y de encuestas por muestreo que básicamente han abordado las migraciones rural-urbanas a ciertas capitales de la república. Respecto a las migraciones internacionales existen tres fuentes: los censos de población norteamericanos, las estadísticas generadas por el Servicio de Inmigración y Naturalización de los Estados Unidos y algunas encuestas llevadas a cabo tanto en México como en su vecino país del Norte. El problema principal de las dos primeras fuentes y de las encuestas realizadas en Norteamérica radica en que la mayoría de los emigrantes mexicanos se encuentran ilegalmente en ese país, por lo que tienden a ocultarse o a mentir cuando son detectados, en cuyo caso los datos así generados adolecen de graves errores, de cobertura y de inexactitud en las características básicas de los migrantes.2 De las encuestas sobre migración a los Estados Unidos efectuadas en México, además de ser pocas se refieren, salvo una, a estudios de caso o fueron realizadas en la frontera norte teniendo como unidad de observación a los mexicanos indocumentados devueltos de Norteamérica por las autoridades de ese país. ${ }^{3}$

Ante este panorama, cuya solución requiere tanto del avance del desarrollo de la parte teórica de la investigación y de la elaboración de una tipología de migraciones, como de un estudio sistemático de los datos existentes, se optó en este primer trabajo por hacer uso, básicamente, de la Encuesta Nacional de Emigración, que constituye la única fuente de información actualmente disponible, donde se generaron estadísticas comparables de migración interna y de emigración internacional, así como de algunos datos de otras fuentes cuyos conceptos resultan similares a los empleados en esta encuesta.

La Encuesta Nacional de Emigración fue realizada por el Centro Nacional de Información y Estadísticas del Trabajo (CENIET) entre diciembre de 1978 y enero de 1979. Esta encuesta se levantó en más de 60,000 viviendas elegidas aleatoriamente a to largo y ancho del territorio de la República Mexicana, $y$ en ellas se entrevistó a sus residentes habituales para obtener información acerca del volumen y las características de los migrantes internos e internacionales. Por constituir los datos de esta encuesta el insumo

\footnotetext{
${ }^{2}$ Para tener una idea sobre la magnirud de la migración legal de mexicanos a Norteamérica en relación a la ilegal, bastan algunos datos generados por el Servicio de Inmigración y Naturalización del Departamento de Justicia de los Estados Unidos, que se refieren al año fiscal de 1977 (octubre de 1976 a septiembre de 1977 ) y que, aunque han sido puestos en tela de juicio, sirven para este propósito: en ese año de 1977 fueron admitidos en esa nación 462,315 personas para residir de manera permanente, de las cuales 44,079 individuos (el $9.5 \%$ ) fueron mexicanos. En cambio, en ese mismo año fueron localizados $1 \% 042,215$ extranjeros deportables, de los que el $91.6 \%$ ( 954,778 personas) fueron de nacionalidad mexicana (la categoría de extranjeros deportables la adquirieron los inmigrantes a los Estados Unidos por haberse internado en ese pais subrepticiamente, sin documentos que les permitan su permanencia legal).

3 Por experiencias directas tenidas en el levantamiento de dos encuestas sobre indocumentados (una efectuada en agosto de 1978 entrevistando a más de 35,000 personas y otra realizada en mayo de 1979 entrevistando a más de 50,000 individuos), así como por la revisión de la coherencia interna de las respuestas obteridas en algunos de los cuestionarios usados en tales ocasiones, hemos concluido que los mexicanos no documentados que son devueltos al país responden verazmente ciertas preguntas (como las relativas a su edad, lugar de origen, estado civil, etc); en cambio,mienten frecuentemente cuando las preguntas se asocian a ciertos aspectos de su migración a los Estados Unidos, como el número de veces que ban cruzado la frontera o la cantidad de ocasiones en que han sido detenidos.
} 
fundamental de estadísticas de este trabajo, se presenta un anexo donde se describen los objetivos y los aspectos más relevantes de la misma, sobre todo los asociados con la "población tipo V" o "flujo ausente de mano de obra", que constituye el grupo de migrantes que principalmente se analiza en este trabajo.

Ahora bien, el hecho de haber elegido los datos de una sola fuente de información, la Encuesta Nacional de Emigración, como los principales insumos para el desarrollo de este trabajo, ${ }^{4}$ no resuelve del todo el problema conceptual y consecuentemente subsiste la dificultad creada por la existencia de varias clases de migraciones, internas e internacionales, que se suceden a veces entre individuos diferentes, a veces entre las mismas personas. Esto es así por que si bien en esta encuesta fueron identificados primero los emigrantes mexicanos a los Estados Unidos y después los migrantes internos que no se habían trasladado a Norteamérica (migrantes internos "puros"), la información relativa a los segundos no ha sido procesada, con lo cual únicamente se dispone de los datos asociados con los migrantes internacionales. Frente a esta situación se optó por diferenciar a los migrantes internacionales en dos conjuntos mutuamente excluyentes -los que tienen antecedentes de migración interna y los que no los tienen- para comparar sus magnitudes y características; ademas, estos dos conjuntos de migrantes se contrastan con datos de otras fuentes sobre migración interna e internacional de la población mexicana.

Esta forma de pretender establecer una serie de similitudes y/o diferencias en las características socioeconómicas, geográficas y demográficassde los migrantes internos y de los migrantes mexicanos a los Estados Unidos, se inicia entonces con la identificación de los antecedentes de migración interna de los emigrantes internacionales, o sea, con la medición del grupo de personas que son tanto migrantes internos como emigrantes internacionales.

La importancia de este inicio radica en que la magnitud de tal conjunto de migrantes internos e internacionales a la vez, en comparación al grupo de emigrantes internacionales "puros", proporciona un primer acercamiento al objetivo, ya que estos dos grupos de migrantes constituyen en conjunto el total de emigrantes mexicanos a los Estados Unidos, con lo que la sola proporción del primer grupo respecto al segundo, al mostrar el tamaño relativo de la intersección entre los fenómenos de migración interna y de migración internacional, da cuenta de la forma en que inciden estos dos fenó-

\footnotetext{
4 Adicionalmente, en este trabajo se emplean como puntos de referencia algunas cifras sobre migrantes y sus características, las cuales han sido generadas madiante otros procesos de generación de informacion. En el caso de las migraciones internas se usan datos del censo de población de 1970 , datos que responden a dos definicio. nea de migración interna: aquella basada en la información sobre lugar de nacimiento y lugar de residencia habitwal al momento como las personas que han tenido al menos un cambio de lugar de residencia habitual. En el caso de las migraciones de mexicanos a los Estados Unidos, se utilizan los resultados de algunas encuestas efectuadas en la frontera norte de México y sobre mexicanos indocumentados devueltos al país por autoridades norteamericanas.
} 
menos migratorios, el interno y el internacional, en una misma población, la mexicana, y orienta, consecuentemente, la determinación de los pasos a seguir en el resto del estudio.

En otras palabras, el grupo constituido por los individuos que son migrantes internos y migrantes internacionales representa a las personas sobre las cuales han actuado tanto los factores que provocan las migraciones internas como los factores que producen las migraciones internacionales. Por ello, si la proporción de este grupo ascendiera al $100 \%$ de los migrantes internacionales (o de los migrantes internos), esto es, si ambos fenomenos migratorios se conformaran por las mismas personas, entonces no cabría la separación de migración interna y de migración internacional, en términos de la búsqueda de las razones que inducen a la población a desplazarse geográficamente y de la formulación de políticas, planes y estrategias que pretendan retener a las personas en sus lugares de origen. En cambio, en el caso del extremo opuesto, o sea, si fuera nulo el grupo de individuos con antecedentes de migración interna y de migración internacional, entonces habría que pensar en la existencia de dos fenómenos migratorios altamente diferenciados, los cuales estarían afectando a dos tipos distintos de mexicanos. Una tercera situación, quizás la de existencia más factible y la que muestre mayor complejidad, se establecería si el grupo de migrantes tanto internos como internacionales alcanzara cualquier porcentaje (mayor que cero y menor que 100) respecto a los conjuntos de migrantes internos "puros" y de emigrantes internacionales "puros"; tal situación induciría a plantear varias lineas de estudio entre las que sobresaldrían aquellas asociadas con las interrogantes: ¿existen en realidad dos fenómenos migratorios distintos, el interno y el internacional?; cexisten en el país tres tipos ajenos de fenómenos migratorios (el que se sucede al interior de las fronteras mexicanas, el que se expresa con desplazamientos desde la república hacia los Estados Unidos y el que se manifiesta a través de dobles movimientos poblacionales, al interior y al exterior de la nación)?; ¿existe una relación necesaria de precedencia entre las migraciones internas y las internacionales?; etc.

De esta manera, en el siguiente apartado se efectúa la medición de la magnitud del grupo de migrantes que son internos e internacionales, empleando para tal efecto distintas definiciones de migración interna, y se compara con el total de migrantes internacionales al que pertenecen; asimismo, se confrontan estas cifras con aquellas asociadas a la migración interna de la población mexicana como un todo, a fin de tener un patrón de referencia sobre los porcentajes de personas que migran. En el último apartado se lleva a cabo el análisis comparativo de algunas características sociales, económicas, demográficas y geográficas de los grupos de migrantes involucrados en el segundo apartado. 


\section{LA MIGRACION INTERNA DE LOS EMIGRANTES MEXICANOS A ESTADOS UNIDOS}

Como fue mencionado, en este apartado se busca medir y analizar la cantidad de migrantes internos que existe entre los emigrantes internacionales $\mathrm{o}$, en otras palabras, se pretende conocer la magnitud del grupo de emigrantes mexicanos a Estados Unidos que tiene antecedentes de migración interna. Para ello, y como también fue señalado, se emplean los datos que sobre emigrantes internacionales han sido generados por la Encuesta Nacional de Emigración (ver anexo). En concreto, se utiliza como información objeto de estudio a las cifras sobre el flujo ausente de mano de obraso emigrantes mexicanos de 15 años y más de edad que, residiendo habitualmente en México, se encontraban en Norteamérica trabajando o buscando trabajo entre mediados de diçiembre de 1978 y mediados de enero de 1979.5

Antes de hacer referencia a los antecedentes de migración interna del flujo ausente de mano de obra, parece conveniente hacer mención de algunas características generales de este grupo de emigrantes internacionales.

La cantidad de personas que componen el flujo ausente de mano de obra asciende a 405 467; esta cifra resulta considerablemente menor al número de mexicanos ilegales en Estados Unidos manejando por el Servicio de Inmigración y Naturalización de los Estados Unidos y por los medios masivos de comunicación; sin embargo, se encuentran en el orden de magnitud de dos estimaciones indirectas obtenidas en sendos trabajos 6 (Robinson establece el rango de 0.37 millones a 2.49 millones de mexicanos indocumentados en Estados Unidos y García lo determina entre 0.48 y 1.22 millones), sobre todo considerando la época en la cual se levantó la Encuesta Nacional de Emigración (diciembre - enero), que es cuando menos demanda de trabajo existe en el sector agrícola norteamericano. $7 \mathrm{La}$ estructura por edades y sexo del flujo ausente resulta típica de las migraciones de caracter temporal: mayoría absoluta del sexo masculino ( 587 hombres por cada 100 mujeres) y concentración de personas en edades activas jóvenes (29.5 años de edad

\footnotetext{
5 A partir de este párrafo denominaremos indistintamente a este grupo de migrantes internacionales como "flujo ausente de mano de obra". "emigrantes internacionales" o "migrantes mexicanos en Estados Unidos".

"Ver: García y Griego, Manuel. El volumen de la migración de mexicanos no documentados en los tistados Unidos: nuevas hipotesis, CENET, México, 1979. Robinson, J. Gitegory. Estimating the Approximate Size of the Megal Alien Population in the United States By Comparative Trend Analysis of Age - Specific Deatli Rates, U. S., Bureau of the Census, Washington, 1979.

7 Adicionalmente, cabe hacer dos aclaraciones referentes al monto del flujo ausente de mano de obra. Por una parte, se tiene conciencia de que el número de integrantes del flujo ausente es una estimación baja del número de emigrantes mexicanos en Fstados Unidos en el periodo de referencia ya que dentro del flujo ausente no se consideraron tres bloques de emigtantes: aquellos con edades menores a los 15 años, aquellos que han emigrado en forma definitiva a los Estados Unidos y aquellos que no ten ían, al momento de las entrevistas, ningún pariente o familiar en México para dar cuenta de su existencia y situación. Por otra parte, se considera que la cifra total del flujo ausente de mano de obra puede estar subestimando la cantidad de nacionales en Norteamérica por el hecho de que algunos componentes de ese flujo quizás no estuvieran en Estados Unidos al efectuarse la encuesta, sino que se encontraban en México, tratando de cruzar la frontera, en ruta hacia el Norte o de regreso a sus hogares.
} 
media y $71.6 \%$ de individuos entre 15 y 34 años de edad cumplida). El nivel educativo de estos migrantes (4.5 años promedio de instrucción) es superior al de la población de 15 y más años de la República Mexicana (3.1 años promedio de instrucción en 1970) e inferior al existente entre los norteamericanos de 25 años y más (en 1970 el número medio de años de instrucción en Estados Unidos fue de 12.1 entre la población blanca y de 10.0 entre los no-blancos). Aproximadamente la mitad del flujo ausente es soltera $(50 \mathrm{o} \%)$, sin embargo, sólo el 30.9 \% de estos emigrantes no tiene de pendientes económicos. Antes de partir a Estados Unidos el $78.0 \%$ estos migrantes tenían ocupación en México, sólo el 3.1 \% estaban desocupados y el $15.8 \%$ eran económicamente inactivos; de los ocupados el $62.9 \%$ laboran en el sector primario. En cuanto al lugar de origen, el 80 \% del flujo ausente de mano de obra reside en ocho entidades federativas (Guanajuato, Jalisco, Chihuahua, Zacatecas, Michoacán, Baja California, Durango y San Luis Potosí), y respecto al destino, el $41.5 \%$ o cruzan la frontera por Tijuana y el $50.9 \%$ se dirige a California. Finalmente y en cuanto a la procedencia urbana rural, puede decirse que los emigrantes son originarios básicamente de zonas rurales (el 78,3\% residen en localidades con menos de 20,000 habitantes).

Estas características relevantes del flujo ausente de mano de obra permiten señalar, en pocas palabras, que la emigración de mexicanos a Estados Unidos es de menor cuantía a la usualmente mencionada por autoridades norteamericanas, es básicamente masculina y de caracter temporal, se produce fundamentalmente entre zonas rurales del centro-norte del país, se dirige principalmente a California, no se origina por falta de empleo sino por la esperanza de obtener mayores ingresos, se sucede entre población joven con niveles educativos superiores a la media nacional, con responsabilidades económicas en México y tanto entre casados como entre solteros. ${ }^{8}$

Así, una vez descrito el perfil general de los emigrantes internacionales que comprenden el flujo ausente, pasamos a la medición de su condición de migración interna. Para tal efecto se utilizan cuatro distintas definiciones de migrantes internos: en la primera se identifica a los migrantes internos cuando son diferentes sus entidades federativas (estados) de nacimiento y de residencia habitual a la fecha de la encuesta; en la segunda, tercera y cuarta se define a los migrantes internos como aquellos que han residido al menos en un lugar distinto de aquel donde se realizaron las entrevistas; es decir, los migrantes internos son las personas que cuando menos han residido habitualmente en dos lugares disímiles. $\mathrm{La}$ diferencia entre estas tres definiciones se encuentra en la unidad geográfica de referencia, el "lugar", que en una definición es la entidad federativa, en otra es el municipio y en la otra es la localidad.

\footnotetext{
8 Un análisis destallado de las características del flujo ausente de mano de obra se presenta en: Zazueta, C y Corona R. Los trabajadores mexicanos en Estados Unidos: primeros resultados de la Encuesta Nacional de limigración (diciembre 1978 - enero 1979) CENIET, México, 1979.
} 


\section{ALGUNOS ASPECTOS CUANTITATTVOS}

De esta manera, se cuenta con cuatro mediciones de la cantidad de los emigrantes internacionales que tienen antecedentes de migración interna, las cuales se presentan en el cuadro 1. Las cuatro mediciones son diferentes porque responden a distintos conceptos, dos de ellos referidos a la migración interna a nivel estado (división político-administrativa mayor), una a la migración interna a nivel municipio (unidad político-administrativa menor), y una más a la migración entre localidades (casi la totalidad de los municipios contienen varias localidades).

Las cifras del cuadro 1 muestran que, de cada 100 emigrantes internacionales 22.2 han cambiado su localidad de residencia, 16.6 han residido en municipios distintos y, entre 9.6 y 13.2 han habitado al menos en dos entidades federativas. Las diferencias entre el número de migrantes internos entre localidades, municipios y estados dan cuenta de la importancia de la unidad territorial de referencia, ya que el aumento de los migrantes al descender la superficie manifiesta desplazamientos, para este caso, entre localidades al interior de municipios y entre municipios dentro de los límites estatales. Es decir, estas cifran señalan que de cada 100 personas que cambiaron de localidad, 74.9 lo hicieron trasladándose a otros municipios, y que, de cada 100 individuos que se desplazaron a otros municipios, unicamente 57.5 rebasaron las fronteras estatales (ver primera columna del cuadro 2).9 En cambio, las diferencias entre las dos estimaciones de la migración interna a nivel estado del flujo ausente evidencian tanto las disparidades conceptuales como problemas en la captación de los datos. ${ }^{10}$

Los porcentajes expuestos en el cuadro 1 adicionalmente exhiben la condición de migración interna del flujo ausente clasificado por sexo; y los porcentajes del cuadro 2 se presentan por regiones de residencia en México (regiones que fueron estructuradas exprofeso para la Encuesta Nacional de Emigrantes de acuerdo a la densidad de emigrantes internacionales). En cuanto a la variable sexo, puede apreciarse que entre los emigrantes internacionales los hombres tienen una ligeramente mayor experiencia de migración interna (por ejemplo, el porcentaje de los emigrantes internacionales que son migrantes internos a nivel localidad es de $22.6 \%$ para el sexo masculino y de 19.9 \% para el femenino). Con respecto a las zonas de residencia en México también se advierten diferencias (bastante más marcadas que las recién se. naladas en relación al sexo), esto es, la distribución de los emigrantes interna.

${ }^{9}$ Cabe señalar que en el cuadro 2 las definiciones de migración interna son aquellas referidas a cuandó nienos un cambio de "lugar" de residencia; es decir, en este cuadro no se incluyen los datos relativos a la migración interna a rivel estatal en función de distintos estados de nacimiento y de residencia actual.

${ }^{10}$ Las dos definiciones usadas para cuantificar la migración a nivel estatal difieren principalmente porque en Ia que identifica a los migrantes internos como aquellos cuyos estados de nacimiento y de residencia actual son distintos no se contempla la llamada "migración de retorno", con lo que su estimación al respectó debería ser más baja que la asociada a la definición de al menos un cambio de entidad federativa de residencia. Sin cunbargo, en este caso resultó mayor la cantidad de migrantes a nivel estado de acuerdo a la definición de entidad de nacimiento distinta a la de residenciz actual $(13.2 \%$ contra $9.6 \%$; ísto sólo puede explicarse por la existencia del relativamente elevado porcentaje de "no especificado" $\{8.1 \%$ o obtenido en las preguntas asociadas a la definición de al menos un cambio de residencia el cual seguramente incluye a personas que efectivamente cambiaron su residencia. 
CUADRO 1. Flujo ausente de mano de obra (emigrantes internacionales de 15 años y más de edad) y población residente en México en 1970 (de 15 años y más) por condición de migración interna de acuerdo a cuatro distintas definiciones de migración interna (porcentajes).

\begin{tabular}{|c|c|c|c|c|c|c|}
\hline \multirow[t]{2}{*}{$\begin{array}{c}\text { Definición y condición de migración } \\
\text { interna } 3\end{array}$} & \multicolumn{3}{|c|}{$\begin{array}{l}\text { Flujo ausente de mano de obra } \\
\text { (migrantes internacionales). }\end{array}$} & \multicolumn{3}{|c|}{$\begin{array}{c}\text { Población residente en México } \\
\text { en } 1970 \text { (de } 15 \text { años y más de } \\
\text { edad). } 2\end{array}$} \\
\hline & $\begin{array}{l}\text { Ambos } \\
\text { sexos }\end{array}$ & Hombres & Mujeres & $\begin{array}{l}\text { Ambos } \\
\text { sexos }\end{array}$ & Hombres & Mujeres \\
\hline $\begin{array}{l}\text { ESTADO DE NACIMIENTO DIFERENTE } \\
\text { ESTADO DE RESIDENCIA ACTUAL. }\end{array}$ & 100.0 & 100,0 & 100.0 & 100.0 & 100.0 & 100.0 \\
\hline $\begin{array}{l}\text { Migrantes internos nivel estado } \\
\text { - No migrantes internos nivel estado } \\
\text { - No especificado. }\end{array}$ & $\begin{array}{r}13.2 \\
86.2 \\
0.6\end{array}$ & $\begin{array}{r}13.5 \\
85.9 \\
0.6\end{array}$ & $\begin{array}{r}11.5 \\
88.2 \\
0.3\end{array}$ & $\begin{array}{l}21.0 \\
79.0\end{array}$ & $\begin{array}{l}20.5 \\
79.5 \\
-\end{array}$ & $\begin{array}{l}21.5 \\
78.5 \\
-\end{array}$ \\
\hline $\begin{array}{l}\text { AL MENOS UN CAMBIO DE ESTADO } \\
\text { DE RESIDENCIA }\end{array}$ & 100,0 & 100.0 & 100.0 & 100.0 & 100.0 & 100.0 \\
\hline $\begin{array}{l}\text { - Migrantes internos nivel estado } \\
\text { - No migrantes internos nivel estado } \\
\text { - No especificado }\end{array}$ & $\begin{array}{r}9.6 \\
82.4 \\
8.1\end{array}$ & $\begin{array}{r}10.0 \\
81.7 \\
8.3\end{array}$ & $\begin{array}{r}6.9 \\
86.5 \\
6.6\end{array}$ & $\begin{array}{l}22.3 \\
77.7 \\
-\end{array}$ & $\begin{array}{l}21.8 \\
78.2 \\
-\end{array}$ & $\begin{array}{l}22.7 \\
77.3 \\
-\end{array}$ \\
\hline $\begin{array}{l}\text { AL MENOS UN CAMBIO DE MUNICIPIO } \\
\text { DE RESIDENCIA }\end{array}$ & 100.0 & 100.0 & 100.0 & - & - & - \\
\hline $\begin{array}{l}\text { - Migrantes internos nivel municipio } \\
\text { - No migrantes internos nivel muni- } \\
\text { cipio } \\
\text { - No especificado }\end{array}$ & $\begin{array}{r}16.6 \\
75.3 \\
8.1\end{array}$ & $\begin{array}{r}16.9 \\
74.8 \\
8.3\end{array}$ & $\begin{array}{r}14.9 \\
78.5 \\
6.6\end{array}$ & $\overline{-}$ & - & $\overline{-}$ \\
\hline $\begin{array}{l}\text { AL MENOS UN CAMBIO DE LOCALIDA } \\
\text { DE RESIDENCLA }\end{array}$ & 100.0 & 100.0 & 100.0 & - & - & - \\
\hline $\begin{array}{l}\text { - Migrantes internos nivel localidad } \\
\text { - No migrantes internos nivel locali- } \\
\text { dad } \\
\text { - No especificado }\end{array}$ & $\begin{array}{r}22.2 \\
70.6 \\
7.2\end{array}$ & $\begin{array}{r}22.6 \\
70.0 \\
7.4\end{array}$ & $\begin{array}{r}19.9 \\
73.9 \\
6.2\end{array}$ & - & 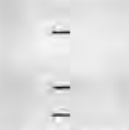 & - \\
\hline
\end{tabular}

1El flujo ausente de mano de obra", que ascendió a 405,467 personas (346,406 hombres y 59,061 mujeres), se define como el conjunto de individuos de 15 años y más de edad que en el momento de las entrevis. tas (realizadas en viviendas dentro de la República Mexicana entre mediados de diciembre de 1978 y mediados de enero de 1979) se encontraban fuera de su residencia habitual en México (lugar de entrevista) porque habian salido a los Estados Unidos a trabajar o buscar trabajo; sobre estas personas informaron los residentes de las viviendas, generalmente parientes.

${ }^{2} \mathrm{La}$ población de 15 años y más de edad residente en México ascendió a 25'938,558 personas 12 '708,253 hombres y $13,230,305$ mujeres).

${ }^{3}$ Los migrantes internos se definen en primer lugar como aquellos cuya entidad federativa de nacimiento es diferente al estado de residencia al momento de las entrevistas; y después, como aquellas personas que han tenido al menos un cambio de localidad de residencia (migrantes internos nivel localidad), de municipio de residencia (migrantes internos nivel municipio), y de entidad federativa de residencia (migrantes internos nivel estado)

FUENTE: R. Corona y C. Ruiz "Migrantes internacionales con y $\sin$. .. op. cit. Cuadro A.1 (cuadro elaborado con datos de la Encuesta Nacional de Emigración y del Censo de Población de 1970). 
CUADRO 2. Flujo ausente de mano de obra, por región de residencia en México, según condición de migración interna.

\begin{tabular}{|c|c|c|c|c|c|c|c|c|c|c|c|c|}
\hline \multirow{2}{*}{$\begin{array}{l}\text { Condición de migración } \\
\text { mterna }\end{array}$} & \multicolumn{7}{|c|}{$\begin{array}{l}\text { Flujo ausente de mano de obra (migrates intern } \\
\text { Región de residencia en México? }\end{array}$} & \multicolumn{5}{|c|}{$\begin{array}{l}5 \text { años y inás de edad) } 1 \text { Porcentajes } \\
\text { Regiöu de residencia en Mexico } 2\end{array}$} \\
\hline & TOTAL. & $\begin{array}{r}\text { Región } \\
1\end{array}$ & $\begin{array}{c}\text { Región } \\
2\end{array}$ & $\begin{array}{l}\text { Región } \\
3\end{array}$ & $\begin{array}{l}\text { Region } \\
4\end{array}$ & $\begin{array}{l}\text { Región } \\
5\end{array}$ & TOTAL & Region & $\begin{array}{l}\text { Región } \\
2\end{array}$ & $\begin{array}{l}\text { Región } \\
3\end{array}$ & Region & $\begin{array}{c}\text { Regibr } \\
5\end{array}$ \\
\hline $\begin{array}{l}\text { TOTAL FLUJO AUSENTEI } \\
\text { MANODE OBRA }\end{array}$ & 100.0 & 100.0 & 100.0 & 100.0 & 100.0 & 100.0 & 100.0 & 10.5 & 46.6 & 25.1 & 14.4 & 3.4 \\
\hline $\begin{array}{l}\text { Migrantes nivel localidad } \\
\text { No migrantes nivel localidad } \\
\text { No especificado }\end{array}$ & $\begin{array}{r}22.2 \\
70.6 \\
7.2\end{array}$ & & $\begin{array}{r}21.3 \\
69.8 \\
8.9\end{array}$ & $\begin{array}{r}9.0 \\
90.1 \\
0.9\end{array}$ & $\begin{array}{r}13.3 \\
80.8 \\
5.9\end{array}$ & $\begin{array}{r}70.7 \\
22.5 \\
6.8\end{array}$ & $\begin{array}{l}100.0 \\
100.0 \\
100.0\end{array}$ & $\begin{array}{r}25.8 \\
4.3 \\
24.3\end{array}$ & $\begin{array}{l}44.5 \\
46.1 \\
57.7\end{array}$ & $\begin{array}{r}10.2 \\
32.0 \\
3.1\end{array}$ & $\begin{array}{l}8.6 \\
16.5 \\
11.7\end{array}$ & $\begin{array}{r}10.9 \\
1.1 \\
3.2\end{array}$ \\
\hline $\begin{array}{l}\text { MIGRANTE } \\
\text { DEL FLUJO } \\
\text { DE OBRA }\end{array}$ & 100.0 & 100.0 & 100,0 & 100.0 & 100.0 & 100.0 & 100.0 & 25.8 & 44,5 & 10.2 & 8.6 . & 10.9 \\
\hline $\begin{array}{l}\text { Migrantes nivel } m \\
\text { No migranter nivc } \\
\text { - No especificado }\end{array}$ & $\begin{array}{r}74.9 \\
21.3 \\
3.8\end{array}$ & $\begin{array}{r}90.9 \\
7.4 \\
1.7\end{array}$ & & $\begin{array}{r}46.5 \\
44.7 \\
8.8\end{array}$ & & $\begin{array}{r}86.2 \\
0.9 \\
12.9\end{array}$ & & $\begin{array}{l}31.3 \\
9.0 \\
11.9\end{array}$ & $\begin{array}{l}414 \\
58.9 \\
26.1\end{array}$ & $\begin{array}{r}6.3 \\
21.4 \\
23.8\end{array}$ & $\begin{array}{r}8.5 \\
10.2 \\
1.2\end{array}$ & $\begin{array}{r}12.5 \\
0.5 \\
37.0\end{array}$ \\
\hline $\begin{array}{l}\text { MIGRANTES N } \\
\text { DEL FLUJO AL } \\
\text { DE OBRA }\end{array}$ & 100.0 & 100.0 & 100,0 . & 100.0 & 100,0 & 100.0 & 100.0 & 31.3 & 41.4 & 6.3 & 8.5 & 12.5 \\
\hline - Mifgrantes nivel estado & $\begin{array}{l}57.5 \\
42.5\end{array}$ & $\begin{array}{l}76.6 \\
23.4\end{array}$ & $\begin{array}{l}31.9 \\
68.1\end{array}$ & $\begin{array}{l}71.4 \\
28.6\end{array}$ & $\begin{array}{l}39.1 \\
60.9\end{array}$ & 100.0 & $\begin{array}{l}100.0 \\
100.0\end{array}$ & $\begin{array}{l}41,6 \\
17.2\end{array}$ & $\begin{array}{l}22.9 \\
66.3\end{array}$ & $\begin{array}{l}7.9 \\
4.3\end{array}$ & $\begin{array}{r}5.8 \\
12.2\end{array}$ & 21.8 \\
\hline
\end{tabular}

1 Ver nota 1. cuadro 1.

2. Las regiones de residencia en México se componen de la siguiente forma

Región 1: Los municipios fronterizos del Norte

Región 2: Los estados de Jalisco, Michoacán, Colima, Guanajuato, parte de los estados de Guerrero y México y el municipio de Ensenada en Baja California.

Región 3: Los estados de Durango, Zacatecas, San Luis Potosi, Querétaro, Nayarit, Aguascalientes y parte de Hidalgo.

Región 4: Parte no fronteriza de los estados de Tamaulipas, Nuevo León, Coahuila, Chihuahua y.Sonora, asi como el estado de Sinaloa.

Región 5: Los estados de Baja California Sur, Campeche, Chiapas, Distrito Federal. Oaxaca, Quintana Roo, Ta basco. Veracruz, Yucatán, Morelos, Puebla, Tlaxcala y parte de los estados de Hidalgo, México y Guerrero.

${ }^{3}$ Ver nota 3, del cuadro 1.

FUENTE: R. Corona y C. Ruiz "Migrantes intemacionales con y $\sin$... op. cit. Cuadro A.2 (cuadro elaborado con datos de la Encuesta Nacional de Emigración). 
cionales conforme a sus regiones dè residencia se altera al considerar solamente al subconjunto de ellos que tienen antecedentes de migración interna: por ejemplo, las regiones 1 y 5 que producen el $13.9 \%$ de los emigrantes internacionales (10.5 \% la región 1 y $3.4 \%$ la región 5$)$, generan el $36.7 \%$, el $43.8 \%$ y el $63.4 \%$ de los mismos pero con experiencia migratoria interna a nivel, respectivamente, de localidad, municipio y estado.

Ahora bien, consideramos que los datos expuestos ponen de manifiesto dos hechos que permiten tanto el planteamiento de una primera hipótesis acerca de la relación entre los fenómenos migratorios interno e internacional, como el establecimiento de la necesidad de profundizar en el estudio sobre este tema de la vinculación, en México; de las migraciones internas con las internacionales.

Por una parte, podemos decir que las cuatro estimaciones del porcentaje de emigrantes internacionales que han migrado internamente, no obstante sus diferencias conceptuales, señalan que la intersección entre los fenómenos de migración interna y de emigración internacional es relativamente pequeña, con excepción de las regiones 1 y 5 . En otros términos, que de los emigrantes mexicanos a Estados Unidos, en general, muy pocos tienen antecedentes de migraciones internas definitivas (solamente uno o dos individuos de cada 10 se encuentran en esta situación), aunque en la zona 1 (la región fronteriza) sucede lo contrario: más de la mitad de sus emigrantes internacionales habían migrado hacia la zona desde otras partes del país, es más, tal hecho cobra relevancia al observar que la experiencia de migración interna del flujo ausente de mano de obra, resulta considerablemente menor a la que tiene la población de 15 años y más del país, (salvo en las regiones 1 y 5 , como se señaló): en 1970, el porcentaje de mexicanos mayores de 14 años que habían cambiado de entidad de residencia superó el $20 \%$; este porcentaje resulta casi el doble del correspondiente a los emigrantes internacionales (también de $15 \mathrm{y}$ más años de edad). 11 Como consecuencia, este primer hecho, que también puede expresarse diciendo que son unos los mexicanos que van a Estados Unidos, otros los que migran internamente y sólo unos cuantos los que realizan ambos desplazamientos, nos induce a establecer la hipótesis de que, en general, son distintos los factores que provocan la movilidad geográfica interna de la población mexicana de aquellos que producen la migración a Norteamérica. Esta hipótesis, que sólo puede demostrarse o rechazarse con el análisis de más información y con su ubicación o no dentro de un esquema teórico que englobe ambos fenómenos migratorios (el interno y el internacional), conlleva implícitamente el establecimiento de algunas consideraciones asociadas a la relación entre estos fenómenos: entre ellas resalta el que, de ser cierta tal hipótesis, no puede pensarse que los desplazamientos de mexicanos a Norteamérica representan

\footnotetext{
11 En el cuadro 1 puede apreciarse que, para 1970,el porcentaje de migrantes internos a nivel estado respecto a la población residente en México (de 15 y más años de edad), fue de $21.0 \%$ de acuerdo a la definición de dis tintas entidádes de nacimiento y residencia, y de $22.3 \%$ conforme a la definición de al menos un cambio de entidad de residencia.
} 
la última etapa de la ruta seguida por los emigrantes internos, o sea, que no es condición necesaria para los emigrantes internacionales haber residido en distintos lugares dentro del territorio nacional.

Por otra parte, consideramos que las diferencias encontradas en los antecedentes de migración interna del flujo ausente de mano de obra, al introducir las variables sexo y lugar de residencia en México, muestran que este grupo de migrantes internos e internacionales a la vez, al no identificarse en primera instancia con el flujo ausente, requiere ser estudiado con mayor detenimiento con el propósito de intentar establecer, al menos, si su perfil socioeconómico es diferente o similar a los migrantes internacionales "puros". Esto permitiría, ulteriormente, el planteamiento, cuando menos, de interrogantes más específicas acerca de la asociación entre las migraciones interna e internacional.

En el siguiente apartado se efectúa un primer análisis comparativo entre algunas características de los emigrantes internacionales "puros" y de los emigrantes internacionales con antecedentes de migración interna.

\section{EL PERFIL SOCIOECONOMICO DE LOS EMIGRANTES INTERNACIO- NALES CON Y SIN EXPERIENCIA DE MIGRACION INTERNA.}

En este último apartado se contrastan los dos grupos en que puede dividirse a los emigrantes internacionales de acuerdo a su condición de migración interna (los que tienen y los que no tienen antecedentes migratorios internos), a través de una serie de indicadores que sintéticamente dan cuenta de su situación socioeconómica. La definición de migración interna elegida para efectuar tal partición del flujo ausente de mano de obra (en emigrantes internacionales "puros" y emigrantes internacionales con experiencia de migración interna) es la que identifica a los migrantes internos como aquellos que han cambiado al menos en una ocasión de localidad de residencia, 12 Los indicadores socioeconómicos que se utilizan para comparar el perfil de los emigrantes internacionales "puros" con los que han migrado internamente, comprenden las siguientes características: sexo (índice de masculinidad), edad (edad media y porcentajes de personas con edades de 15 a 24 años), escolaridad (número medio de años de estudios y porcentajes de individuos sin instrucción y con más de 9 años de instrucción), estado civil (porcentajes de solteros, casados y en unión libre, y de otros estados civiles), zona urbana o rural de residencia en México (porcentaje de personas que viven en localidades menores y mayores de 20,000 habitantes, clasificado por regiones), responsabilidad económica (número medio de dependientes economicos en el país y porcentaje de individuos sin dependientes), ayuda

\footnotetext{
12De acuendo a esta definición, el total de 405,467 emigrantes internacionales (o flujo ausente de mano de obra) se clasifica en 286,211 emigrantes internacionales "puros", 89,960 emigrantes internacionales con antecedentes de migración interna a nivel localidad y 29,296 emigrantes internacionales que no especificaron su condición de mgración interna.
} 
económica de otros familiares (número medio de contribuyentes al ingreso familiar y porcentaje de individuos que son el único sosten económico de la familia) y características de ocupación en México antes de salir a Estados Unidos (condición de actividad económica, porcentajes de ocupados en los sectores primario y de servicios, y porcentajes de ocupados como obreros o empleados y como trabajadores familiares no remunerados). Los índices de masculinidad, que se elaboraron por regiones, se muestran en el cuadro 3 ; $y$ el resto de indicadores se presentan en el cuadro 4 clasificados por sexo.

En cuanto a las dos variables demográficas contempladas (sexo y edad) y al origen urbano o rural del flujo ausente de mano de obra,se observa lo siguiente. La anotada mayoría absoluta de hombres entre el flujo ausente de mano de obra ( 5.9 varones por cada hembra), aunque prevalece en ambos tipos de emigrantes internacionales, se eleva para los migrantes internos (6.6 hombres por mujer) y disminuye para los que no tienen desplazamientos domésticos (5.6 hombres por mujer). La estructura por edades, joven de por sí para el flujo ausente de mano de obra, especialmente entre el sexo femenino, varía considerablemente de acuerdo a la condición de migración interna, pero manteniéndose las diferencias entre sexos: los emigrantes internacionales con migración interna exhiben una distribución por edades más vieja que la correspondiente a los migrantes internacionales "puros" (la edad media de los primeros, 33.6 años, es 6.1 años más elevado que la de los segundos, los cuales concentran a la mitad de sus integrantes en el grupo de 15 a 24 años). El origen principalmente rural de los emigrantes internacionales en conjunto ( 21.7 \% viven en localidades con 20,000 y más habitantes) se altera notablemente conforme a los antecedentes de movilidad geográfica interna, residiendo en áreas urbanas casi la mitad $(46.3 \%$ ) de aquellos con migración doméstica y únicamente uno de cada $10(11.0 \%$ omigrantes internacionales "puros" residen en estas mismas zonas urbanas. Esta procedencia urbana diferencial de los emigrantes internacionales conforme su experiencia de migración interna se observa también en las cuatro regiones que aportan el $96.6 \%$ del flujo ausente, en todos los hombres y en la mayoría de las mujeres.

Respecto a las características sociales (escolaridad y estado civil), los indicadores del cuadro 4 ponen de manifiesto, por una parte, que el nivel educativo de los emigrantes internacionales con y sin antecedentes de migración interna es ligeramente mayor entre los últimos ( 4.3 y 4.5 años promedio de instrucción), y con marcadas diferencias en su composición: entre los emigrantes internacionales "puros", su escolaridad se concentra alrededor de su propio número medio de años de instrucción (solamente el $7.5 \%$ y $4.0 \%$ de estos emigrantes se encuentran, respectivamente, en las categorías "sin instrucción" y "con más de nueve años de escolaridad"); mientras que entre el flujo ausente con antecedentes de migración interna el nivel educativo se dispersa, habiendo un $16.2 \%$ o que no han recibido enseñanza formal y un $7.6 \%$ que han cursado $10 \mathrm{y}$ más años de instrucción. Por otra parte, se advierten grandes disparidades en el estado civil de uno u otro grupo de emigrantes internacionales: el perfil de los que tienen migraciones 


\section{CUADRO 3. Flujo ausente de mano de obra, por región de residencia en Mé- xico, según sexo y condición de migración interna a nivel lo- calidad.}

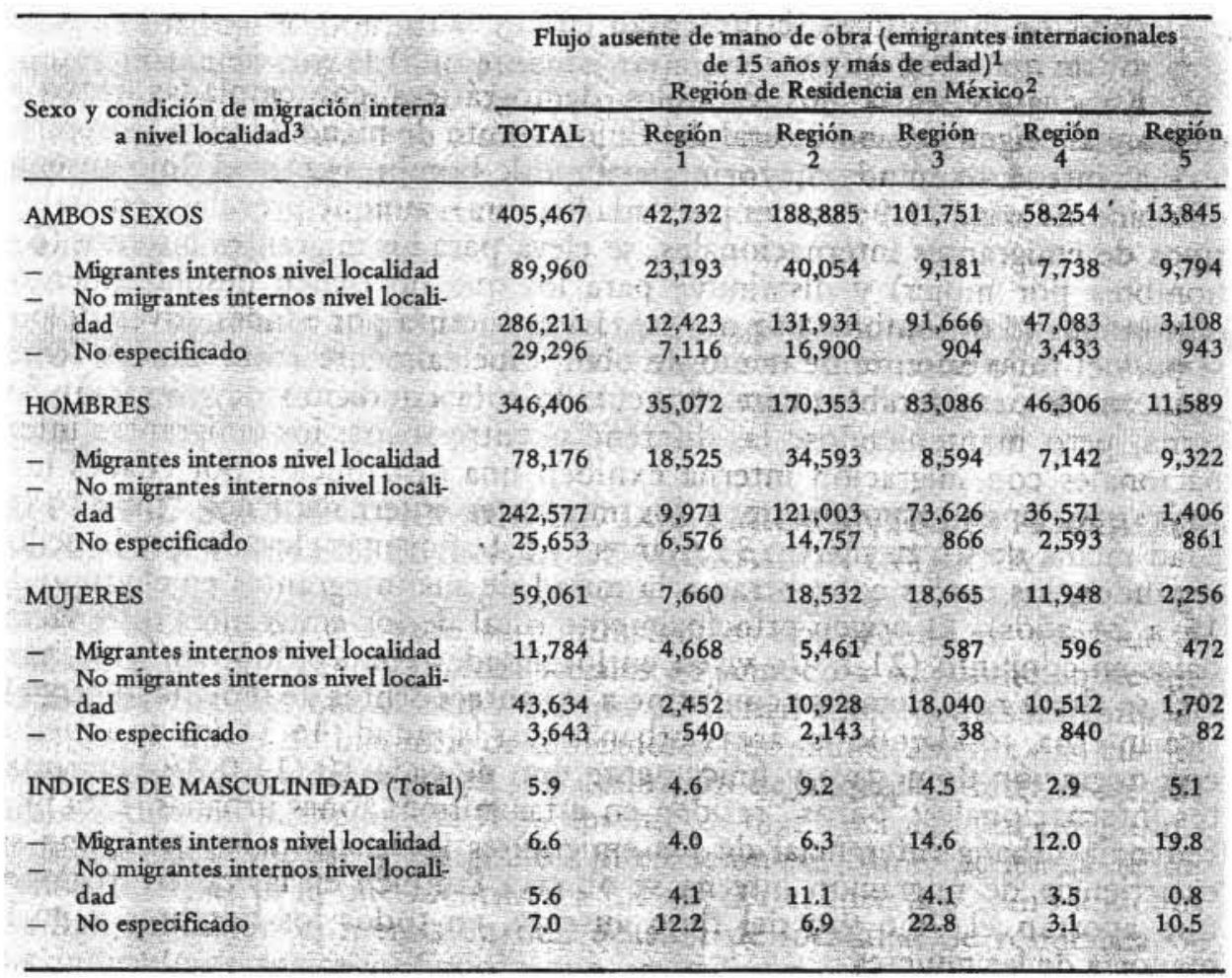

1 Ver nota 1 del cuadro 1.

2 Ver nota 2 del cuadro 2.

${ }^{3}$ Ver nota 3 del cuadro 2.

FUENTE: R. Corona y C. Ruiz "Migrantes intemacionales con y sin... op. cit. Cuadros A.2, A.3 y A.4 (Cuadros elaborados con datos de la Encuesta Nacional de Emigración). 
CUADRO 4. Flujo ausente de mano de obra, por condición de migración interna a nivel localidad y sexo, según algunos indicadores de características socioeconómicas y demogrảficas.

\begin{tabular}{|c|c|c|c|c|c|c|c|c|c|}
\hline \multirow{3}{*}{$\begin{array}{l}\text { Características sociales, } \\
\text { demográficas y económicas }\end{array}$} & \multicolumn{9}{|c|}{$\begin{array}{l}\text { Flujo ausente de mano de obra (enigrantes internacionales de } 15 \text { años } \\
\qquad y \text { más de edad): porcentajes } 1\end{array}$} \\
\hline & \multirow[b]{2}{*}{$\begin{array}{l}\text { Ambos } \\
\text { sexos }\end{array}$} & \multirow[b]{2}{*}{ Hombres: } & \multirow[b]{2}{*}{ Mujeres } & \multicolumn{3}{|c|}{$\begin{array}{l}\text { Migrantes internos }{ }^{2} \\
\text { nivel localidad }\end{array}$} & \multicolumn{3}{|c|}{$\begin{array}{l}\text { No migrantes internos } \\
\text { nivel localidad }\end{array}$} \\
\hline & & & & $\begin{array}{l}\text { Ambos } \\
\text { sexos }\end{array}$ & Hombres & Migjeres & $\begin{array}{l}\text { Ambos } \\
\text { sexos }\end{array}$ & Hombres: & Mrijeres \\
\hline $\begin{array}{l}\text { EDAD } \\
-\quad \% \text { de } 15 \text { a } 24 \text { años } \\
\text { - Edad media }\end{array}$ & $\begin{array}{l}45.9 \\
29.5\end{array}$ & $\begin{array}{l}44.6 \\
29.8\end{array}$ & $\begin{array}{l}53.1 \\
28.0\end{array}$ & $\begin{array}{l}36.7 \\
33.6\end{array}$ & $\begin{array}{l}36.1 \\
33.9\end{array}$ & $\begin{array}{l}41.1 \\
31.8\end{array}$ & $\begin{array}{l}50.5 \\
27.5\end{array}$ & $\begin{array}{l}49.2 \\
27.7\end{array}$ & $\begin{array}{l}57.3 \\
26.6\end{array}$ \\
\hline \multicolumn{10}{|l|}{ BSCOLARIDAD } \\
\hline $\begin{array}{l}\text { - } \% \text { sin instrucción } \\
-\quad \% \text { con más de } 9 \text { años de instrucción } \\
-\quad \text { Número medio de años de estudio }\end{array}$ & $\begin{array}{r}10.4 \\
5.3 \\
4.5\end{array}$ & $\begin{array}{r}11.1 \\
4.7 \\
4.4\end{array}$ & $\begin{array}{l}6.1 \\
9.0 \\
5.1\end{array}$ & $\begin{array}{r}16.2 \\
7.6 \\
4.3\end{array}$ & $\begin{array}{r}17.2 \\
6.3 \\
4.2\end{array}$ & $\begin{array}{r}10.1 \\
16.3 \\
4.8\end{array}$ & $\begin{array}{l}7.5 \\
4.0 \\
4.5\end{array}$ & $\begin{array}{l}8.2 \\
3.6 \\
4.4\end{array}$ & $\begin{array}{l}3.6 \\
6.1 \\
5.3\end{array}$ \\
\hline \multicolumn{10}{|l|}{ ESTADO CIVIL } \\
\hline - \% solteros & 50.2 & 49.3 & 55,3 & 32.8 & 31.5 & 41.2 & 58.1 & 57.4 & 61.5 \\
\hline - \% casados y unión libre & 47.9 & 50.0 & 35.6 & 64.3 & 67.6 & 42.4 & 40.6 & 42.2 & 31.9 \\
\hline - $\%$ viudos, separados y divorciados. & 19 & 0.7 & 91 & 2.9 & 0.9 & 16.4 & 1.3 & 0.4 & 6.6 \\
\hline \multicolumn{10}{|l|}{ RESIDENCIA URBANA O RURAL 3} \\
\hline$\%$ habitan zonas urbanas & 21.7 & 20.2 & 30.4 & 46.3 & 45.7 & 50.4 & 11.0 & 9.0 & 21.9 \\
\hline Ent & 79.0 & 79.1 & 78.9 & 84.6 & 85.6 & 80.6 . & 64.2 & 59.2 & 81.7 \\
\hline En region 2 & 17.8 & 15.8 & 36.1 & 35.5 & 36.8 & 26.9 & 9.9 & 7.8 & 33.3 \\
\hline En región 3 & 2.0 & 2.2 & 1.0 & 11.0 & 11.8 & - & 0.8 & 0.7 & 11. \\
\hline En región 4 & 220. & 21.7 & 23.2 & 40.7 & 40.5 & 43.5 & 16.3 & 15.5. & 19.0 \\
\hline En region 5 & 43.5 & 32.7 & 99.0 & 39.7 & 36.9 & 951 & 61.6. & 15.1 & 100.0 \\
\hline \multicolumn{10}{|l|}{ DEPENDIENTES ECONOMICOS } \\
\hline - $\%$ ningün dependiente & 30.9 & 26.7 & 55.4 & 18.7 & 14.2 & 48.8 & 35.6 & 31.6. & 57.8 \\
\hline - Número medio de dependientes & 3.2 & 3.5 & 2.0 & 3.9 & 4.4 & 2.2 & 3.0 & 3.2 & 1.9 \\
\hline \multicolumn{10}{|l|}{$\begin{array}{l}\text { CONTRUBUYENTES AL } \\
\text { INGRESO FAMILIAR }\end{array}$} \\
\hline - \% ninguna persona que contribuye & 9.3 & 9.6 & 7.0 & 13.8 & 15.5 & 2.5 & 79 & 7.8 & 8.5 \\
\hline - Número medio de contribuyentes & 1.8 & 1.8 & 2.2 & 1.6 & 1.5 & 2.2 & 1.9. & 1.2 & 2.2 \\
\hline \multicolumn{10}{|l|}{$\begin{array}{l}\text { CARACTERISTICAS } \\
\text { ECONOMICAS }\end{array}$} \\
\hline - \% PEA ocupada & 78.0 & 86.6 & 27.6 & 77.9 & 84.9 & 31.6 & 78.0 & 873 & 26.3 \\
\hline - \% PEA desocupada & 3.1 & 3.4 & 1.4 & 2.7 & 2.9 & 1.4 & 3.3 & 3.7 & 0,7 \\
\hline$\% \mathrm{P}$ & 15.8 & 6.8 & 68.6 & 14.1 & 71 & 60.6. & 16.5 & 6.6 & 21.6 \\
\hline - \% no especificado & 31 & 32 & 2.4 & 53 & 5.1 & 6,4 & 2.2 & 2.4 & 14 \\
\hline \multicolumn{10}{|l|}{ CARACTERISTICAS ECONOMICAS } \\
\hline RADOS & & & & & & & & & \\
\hline$\%$ ocupados en sector a gropecuario & 62.9 & 65.8 & 94 & 43.6 & 45,1 & 16.0 & 69.4 & 72.7 & 72 \\
\hline - 86 ocupados eri sector servictos & 9.7 & 5.6 & 470 & 11.2 & 93 & 45.0 & $6.0 \%$ & 3.9. & 45.6 \\
\hline - \% obreroso empleados. & 27.0 & 23.9 & 83.2 & 42.6 & 41.0 & 71.1 & 21.5 & 17.9 & 88.7 \\
\hline - \% trabajador familiar no remunerad & 23.0 & 24.2 & 0.2 & 11.8 & 124 & 15 & 27.6 & 29.0 & 1.1 \\
\hline
\end{tabular}

${ }^{1}$ Ver nota 1 del cuadro 1.

${ }^{2}$ Ver nota 3 del cuadro 1 .

${ }^{3}$ Las zonas urbanas son aquellas localidades de 20,000 o más habitantes, las zonas rurales son aquellas localida. des con inenos de 20,000 habitantes; en cuanto a la regionalización ver nota 2 del cuadro 2 .

FUENTE: R. Corona y C. Ruiz "Migrantes intemacionales con y sin. . op cit. Cuadros A.9, A.10 y A.13 a A.30 (cuadros elaborados con datos de la Encuesta Nacional de Emigración). 
internas los ubica como personas en su mayoría con familia propia $(32.8 \%$ solteros, $64.3 \%$ casados o unidos libremente y $2.9 \%$ viudos, separados o divorciados); en cambio, el estado civil de los emigrantes internacionales "puros" identifica a tres de cada cinco de ellos como hijos de familia $(58.1 \%$ solteros, $40.6 \%$ casados o en unión libre y $1.3 \%$ viudos, separados o divorciados). Estas diferencias se presentan en igual sentido en los dos sexos, aunque menos marcadas en el femenino, respecto al cual cabe resaltar el elevado porcentaje de viudas, divorciadas y separadas en ambas clases de emigrantes internacionales, en especial en la que considera experiencia de migración interna, donde tal porcentaje asciende a $16.4 \%$.

Las características económicas, medidas a través de los indicadores señalados, pueden dividirse en dos bloques; uno referente a las actividades económicas del flujo ausente, y otro, asociado más bien a sus necesidades de ingresos.

En relación a las actividades económicas y en primer lugar en cuanto a la condición de actividad en México antes de desplazarse a Norteamérica,

no se aprecian distinciones significativas entre ambos tipos de emigrantes internacionales (77.9 \% y $78.9 \%$ de ocupados, $2.7 \%$ y $3.3 \%$ de desocupados y, $14.1 \%$ y $16.5 \%$ o de económicamente inactivos, respectivamente para emigrantes internacionales con y sin migración interna). En segundo lugar, respecto a la rama de actividad y a la posición en el trabajo de los ocupados, podemos ver que existen relevantes diferencias entre los emigrantes internacionales con y sin migración interna: el $43.6 \%$ del flujo ausente ocupado con experiencia de migración doméstica laboraba en el sector agropecuario y el 69.4 \% de los emigrantes internacionales "puros" se encontraba en la misma situación; el porcentaje de ocupados en servicios fue de $11.2 \%$ y de $6.0 \%$ para el primero y el seguno tipo de migrantes, los porcentajes de obreros y empleados y de trabajadores familiares no remunerados llegaron a $42.6 \%$ y $11.8 \%$ para aquellos con experiencia de migración interna, y a 21.5 \% y $27.6 \%$ o para los otros emigrantes internacionales. Estos porcentajes muestran que los emigrantes internacionales "puros" realizan, en México, actividades mucho más relacionadas con el agro que los migrantes con desplazamientos internos e internacionales, lo cual evidencia una situación de la misma índole que la expresada por el origen urbano-rural de los migrantes.

Por su parte, los indicadores económicos vinculados a las necesidades de ingresos revelan que los emigrantes internacionales con desplazamientos geográficos internos se enfrentan a requerimientos económicos mayores que aquellos sin tales desplazamientos. Mientras en promedio cada uno de los primeros tiene que mantener a 3.9 personas recibiendo la ayuda de 1.6 individuos, cada uno de los segundos sostiene a 3.0 gentes con la colaboración de 1.9 personas. 
Así pues, tomando en conjunto los señalamientos de los diversos indicadores empleados puede decirse que los mismos establecen dos situaciones de los emigrantes internacionales "puros" frente a los emigrantes internacionales que previamente habían migrado en el interior del país. En primer término, que las diferencias en sus perfiles socioeconómicos permiten establecer que se trata de dos grupos distintos de personas. En segundo término, que las mismas diferencias apuntan todas en el sentido de identificar a los emigrantes internacionales con antecedentes de migración doméstica como un conjunto de individuos con necesidades económicas más apremiantes, quizás con mayores dificultades para encontrar el empleo que las soluciones, 13 (por su edad relativamente más elevada y por su menor escolaridad), como lo señala el simple hecho de que antes de trasladarse a los Estados Unidos intentaron sobrevivir decorosamente en otras partes de la república (principalmente en áreas urbanas).

\section{CONCLUSIONES}

En análisis de los datos disponibles sobre la proporción de los emigrantes mexicanos a Estados Unidos que tienen antecedentes de migración interna (Apartado 2), y acerca de las características socioeconómicas y demográficas, tanto de los emigrantes internacionales con experiencia de migración doméstica, como de aquellos emigrantes internacionales sin tales antecedentes (Apartado 3), permiten concluir, que en México, y haciendo a un lado la zona fronteriza, los fenómenos de emigración internacional y de migración interna definitiva afectan, en términos generales, a distintos grupos de población. ${ }^{14}$ Asimismo, los datos señalan que el relativamente reducido grupo de emigrantes internacionales con migraciones internas previas, exhibe características demográficas y socioeconómicas distintas al conjunto de emigrantes internacionales "puros"; distintas en el sentido de ubicarlos en una parte más baja de la escala social y económica.

Las anteriores conclusiones, que representan los dos hechos más relevantes entre los mostrados por las cifras manejadas, refuerzan la idea de continuar la realización de estudios descriptivos como este trabajo, acompañados de investigaciones de naturaleza teorica sobre la relación entre los fenómenos migratorios interno e internacional. Es decir, las conclusiones de este trabajo implican, entre otras cosas, que los emigrantes internacionales no migran en su gran mayoría en primera instancia a otras partes del país antes de cruzar la frontera (salvo en el caso de la misma zona fronteriza), y que aquellas personas con ambas clases de migraciones efectúan la internacional después de no poder solucionar sus necesidades y satisfacer sus aspiraciones

\footnotetext{
${ }^{13}$ Conviene destacar que, desgraciadamente, entre los datos disponibles no se encontró ninguno relativo al fenómeno del subempleo, fenómeno de altas proporciones en el pais y cuyas cifras respecto a los emigrantes internacionales hubieran aumentado considerablemente los elementos de juicio manejados en este trabajo.

$14 \mathrm{En} \mathrm{la} \mathrm{frontera} \mathrm{norte} \mathrm{el} \mathrm{fenómeno} \mathrm{se} \mathrm{sucede} \mathrm{en} \mathrm{sentido} \mathrm{inverso:} \mathrm{en} \mathrm{ese} \mathrm{lugar} \mathrm{la} \mathrm{migración} \mathrm{interna} \mathrm{y} \mathrm{la} \mathrm{interna-}$ cional se mezcian de tal forma que el $54.2 \%$ de sus emigrantes a Estados Unidos eran inmigrantes de otras partes del país.
} 
a través de sus desplazamientos internos; entonces, de llegar a verificar simplemente los señalamientos expresados en las conclusiones mencionadas, habría que pensar en profundizar acerca de las causas diferenciales que motivan uno y otro movimiento migratorio, en abrir las categorías teóricas usadas en la mayoría de los trabajos sobre ambos fenómenos en México, tanto para esclarecer las explicaciones de la existencia de ambas clases de migraciones en el contexto del desarrollo socioeconómico nacional y de su vinculación con el de Norteamérica, como en el ámbito de la política demográfica mexicana, para una determinación más adecuada y apegada a la realidad, de los planes, programas y acciones tendientes a retener a los migrantes en sus lugares de origen y reubicar y reorientar las corrientes migratorias de mexicanos.

\section{A N E X O}

\section{LA ENCUESTA NACIONAL DE EMIGRACION}

La Encuesta Nacional de Emigración forma parte del proyecto "Encuesta Nacional de Emigración a la Frontera Norte del País y a los Estados Unidos" (ENEFNEU), que realiza desde abril de 1977 el Centro Nacional de Información y Estadísticas del Trabajo (CENIET) de la Secretaría del Trabajo y Previsión Social. Este proyecto, ENEFNEU, se compone por cuatro encuestas, siendo la tercera en llevarse a cabo la Encuesta Nacional de Emigración, que es una encuesta por muestreo realizada del 11 de diciembre de 1978 al 7 de enero de 1979 entre los residentes de más de 60,000 viviendas en 115 localidades de la República Mexicana. Las otras tres encuestas fueron levantadas en la frontera norte del país en octubre-noviembre de 1977, agosto de 1978 y mayo de 1979 y tuvieron como unidad de observación a trabajadores mexicanos no documentados devueltos a México por autoridades norteamericanas. Estas tres encuestas, donde se entrevistaron aproximada y correspondientemente a 10,000 , a 30,000 y 50,000 migrantes, tuvieron como propósitos generales conocer algunas características socioeconómicas de los emigrantes mexicanos ilegales localizados y devueltos al país por el personal de la Patrulla Fronteriza (Border Patrol) de los Estados Unidos y alimentar un modelo demográfico para calcular el monto de emigrantes nacionales a Norteamérica. Los objetivos especiales de estas encuestas sobre indocumentados fueron, para las dos primeras, obtener las bases cuantitativas indispensables para la elaboración del marco muestral de la Encuesta Nacional de Emigracion (densidad de emigrantes por municipio) y entrenar a los supervisores de la misma; y para la última, su objetivo especial fue determinar la posibilidad de crear un sistema de encuestas de frontera para conocer el monto y características de los emigrantes mexicanos a Estados Unidos.

Los objetivos de la Encuesta Nacional de Emigración fueron: determinar el flujo migratorio de mano de obra mexicana a los Estados Unidos y sus principales características; determinar la migración interna de la población mexicana, con énfasis en la migración a la frontera norte del país y 
sus principales características, y determinar el volumen de la población mexicana no migrante y sus principales características.

Para cumplir con estos propósitos, en la Encuesta Nacional de Emigración se aplicaron cuatro instrumentos de captación de datos: en el primero, denominado boleta, se obtuvo información socioeconómica y demográfica de todos los residentes de las viviendas y se identificó a las personas con antecedentes migratorios (tanto internos como internacionales); en los otros tres instrumentos de captación, llamados cuestionarios, se captaron datos socioeconómicos y demográficos más desagregados y sobre la historia migratoria de las personas identificadas como migrạntes en la boleta.

Los migrantes fueron localizados en la boleta de la siguiente manera: primero se díferenció a los residentes de cada vivienda en menores de 15 años $\mathrm{y}$ en personas con 15 y más años de edad; después se reconoció, de entre los individuos de 15 años y más, a los migrantes internacionales dividéndolos en dos tipos (los ausentes y los de retorno) gracias al uso de dos definiciones complementarias y excluyentes; a continuación se partió el grupo de los no migrantes internacionales mayores de 14 años en migrantes internos y no migrantes internos. Una vez clasificada a la población de 15 y más años de edad en los cuatro grupos mencionados, se aplicaron los tres cuestionarios (siguiendo un procedimiento preestablecido de selección): el primero para migrantes internacionales ausentes, el segundo para los migrantes internacioles de retorno y el tercer cuestionario tanto para los migrantes internos como para los no migrantes internos.

De acuerdo a este proceso de identificación y a las definiciones usadas los cuatro tipos de población sobre quienes se pidió informacion en los tress cuestionarios quedaron constituidos como sigue:

1) Flujo ausente de mano de obra: personas de 15 años y más de edad residenes habituales de las viviendas seleccionadas y de los cuales sus familiares declararon que no se encontraban en sus lugares por haberse ido a Estados Unidos a trabajar o a buscar trabajo.

2) Flujo de mano de obra de retorno: individuos de 15 años y más de edad que, estando presentes en su residencia habitual, habían ido a trabajar o a buscar trabajo a los Estados Unidos entre el primero de enero de 1974 y la fecha de la entrevista (diciembre de 1978 - enero de 1979).

3) Migrantes internos: personas mayores de 14 años que, no siendo migrantes internacionales (flujo ausente de mano de obra o flujo de mano de obra de retorno) han radicado fuera del municipio de residencia actual (donde se realizaron las entrevistas) en algún otro municipio de la República Mexicana.

4) Población no migrante: personas de 15 años y más no incluidas dentro de ninguno de los tres tipos de población anteriores. 
Así la Encuesta Nacional de Emigración contempló en sus diferentes instrumentos de captación las características de la pobalción del país, tanto en aspectos sociales demográficos y económicos, como en temas asociados con la historia migratoria de las personas identificadas como migrantes. Los cuestionarios aplicados, aunque buscando todos obtener características comparables de naturaleza socioeconómica y demográfica, difieren entre sí por las preguntas relativas a los antecedentes migratorios; es decir, en el cuestionario usado para los migrantes internos y para la población no migrante, no se incluyeron preguntas sobre migración internacional; en cambio, en los cuestionarios referentes a los migrantes internacionales se hizo referencia especial a la forma y lugar de entrada a los Estados Unidos, lugar de permanencia, condición de trabajo y migratoria en ese país, etc. Las diferencias entre los cuestionarios correspondientes a los dos tipos de migrantes internacionales se encuentran en que uno, el asociado al flujo de mano de obra de retorno contiene preguntas más detalladas sobre la historia miindividuos del flujo ausente de mano de obra, solamente aborda aspectos generales de la emigración internacional y de la posible migración interna de estos individuos. Esta diferencia en el contenido de los cuestionarios de los dos tipos de migrantes internacionales se debe a que, mientras los integrantes del flujo de mano de obra de retorno se encontraban presentes y podían contestar preguntas detalladas, los migrantes pertenecientes al flujo ausente de mano de obra, al encontrarse precisamente en Estados Unidos, no podían ser entrevistados, con lo cual las preguntas debían ser mínimas y con posibilidad de ser respondidas por sus familiares (los habitantes de las viviendas elegidas).

Como puede apreciarse por lo expuesto, la Encuesta Nacional de Emigración se presenta como una excelente fuente de datos para la investigación del fenómeno migratorio, tanto interno como internacional; sin embargo, a la fecha solamente se han generado algunas tabulaciones referidas al flujo ausente de mano de obra. Respecto al objetivo del presente trabajo, indagar aspectos de la relación entre la migración interna y la internacional, se utilizan los pocos datos disponibles, empleando para ello la información que sobre antecedentes de migración interna se captó en los cuestionarios aplicados al flujo ausente de mano de obra. 